\title{
Advances in Functional Magnetic Resonance Imaging: Technology and Clinical Applications
}

\author{
Bradford C. Dickerson \\ Gerontology Research Unit, Alzheimer's Disease Research Center, and Athinoula A. Martinos Center for Biomedical Imaging, \\ Department of Neurology, Massachusetts General Hospital; Memory Disorders Unit, Division of Cognitive and Behavioral \\ Neurology, Department of Neurology, Brigham \& Women's Hospital; Department of Neurology, Harvard Medical School, \\ Charlestown, Massachusetts 02129
}

\begin{abstract}
Summary: Functional MRI (fMRI) is a valuable method for use by clinical investigators to study task-related brain activation in patients with neurological or neuropsychiatric illness. Despite the relative infancy of the field, the rapid adoption of this functional neuroimaging technology has resulted from, among other factors, its ready availability, its relatively high spatial and temporal resolution, and its safety as a noninvasive imaging tool that enables multiple repeated scans over the course of a longitudinal study, and thus may lend itself well as a measure in clinical drug trials. Investigators have used fMRI to identify abnormal functional brain activity during task performance in a variety of patient populations, including those with neurodegenerative, demyelinating, cerebrovascular, and other neurological disorders that highlight the potential utility of fMRI in both basic and clinical spheres of research. In
\end{abstract}

addition, fMRI studies reveal processes related to neuroplasticity, including compensatory hyperactivation, which may be a universally-occurring, adaptive neural response to insult. Functional MRI is being used to study the modulatory effects of genetic risk factors for neurological disease on brain activation; it is being applied to differential diagnosis, as a predictive biomarker of disease course, and as a means to identify neural correlates of neurotherapeutic interventions. Technological advances are rapidly occurring that should provide new applications for fMRI, including improved spatial resolution, which promises to reveal novel insights into the function of fine-scale neural circuitry of the human brain in health and disease. Key Words: Alzheimer's disease, mild cognitive impairment, plasticity, functional magnetic resonance imaging.

\section{INTRODUCTION}

Whether or not there is a discrete, macroscopically visible structural lesion, many neurological disorders are characterized by abnormal brain function. The neurologist frequently relies on behaviorally observable abnormalities during the performance of a task in the clinical evaluation of patients. Although a number of diagnostic testing modalities provide information on resting brain function, including electroencephalography, positron emission tomography (PET), and single photon emission computed tomography, there are relatively few tools available to probe regional brain activity during task performance. One of the most readily available tools at present is functional MRI (fMRI).

For approximately the past decade, fMRI has under-

Address correspondence and reprint requests to: Bradford $\mathrm{C}$. Dickerson, MD, Gerontology Research Unit, Massachusetts General Hospital, 149 13th Street, Suite 2691, Charlestown, MA 02129. E-mail: bradd@nmr.mgh.harvard.edu. gone rapid growth in its use as a technique to study functional brain abnormalities in patients with neurological diseases. The use of fMRI in studies of patients with neurodegenerative diseases illustrates a number of applications of this technology. Beyond traditionally recognized neurodegenerative diseases, studies in neurodevelopmental disorders (such as autism ${ }^{1}$ ) and in neuropsychiatric disorders (such as schizophrenia ${ }^{2}$ or individuals at genetic risk for schizophrenia ${ }^{3}$ ) have also begun to provide fundamental insights into the neural processing abnormalities that characterize these disorders. Functional MRI has also revealed novel observations in investigations of stroke and other cerebrovascular diseases, multiple sclerosis, head injury, and other neurological disorders. Ultimately, fMRI may prove to be useful as an imaging biomarker for use in the development of neurotherapeutic interventions for many of these disorders. ${ }^{4}$ This review will summarize data primarily from investigations of Alzheimer's disease (AD) and mild cognitive impairment (MCI) to illustrate potential clinical neurological applications of fMRI, most of 
which have parallels in other neurological disorders. Finally, this review will briefly touch on some recent technical advances in fMRI, pushing the frontiers of spatial resolution, which may enable novel future clinical applications.

\section{fMRI: ADVANTAGES AND DISADVANTAGES}

Because the functional neuroimaging tools assess inherently dynamic processes that may change during short-time intervals in relation to a host of factors, these measures have unique characteristics that may offer both strengths and weaknesses as potential biomarkers of neurological disease. Functional neuroimaging measures may be affected by transient brain and body states at the time of imaging, such as arousal, attention, sleep deprivation, sensory processing of irrelevant stimuli, or the effects of substances with pharmacological CNS activity. Imaging measures of brain function may also be more sensitive than structural measures to constitutional or chronic differences between individuals, such as genetics, intelligence or educational level, learning, mood, or medication use. Although these may be effects of interest in certain experimental settings, they need to be controlled when the focus is on disease-related changes and differences between subject groups or within individuals followed over a period of time.

Among functional neuroimaging techniques, fMRI has many potential advantages in studying neurological patients, because it is a noninvasive imaging technique that does not require the injection of contrast agent. This technique can be repeated many times during the course of a longitudinal study; therefore, it has potential as a measuring tool for use in clinical drug trials. It has relatively high spatial and temporal resolution, and the use of event-related designs enables the hemodynamic correlates of specific behavioral events to be measured. ${ }^{5}$

However, there are significant challenges to performing fMRI studies in neurologically impaired patients. This technique is particularly sensitive to even small amounts of head motion. Differences in task performance between patient and control groups complicate data interpretation. ${ }^{6}$ Finally, it is critical to complete further reliability experiments if fMRI is to be used in longitudinal or pharmacological studies. Although there are now a few studies of fMRI test-retest reliability in young subjects, ${ }^{7-9}$ reproducibility studies are only beginning to be performed in neurological patients.

\section{CLINICAL APPLICATIONS OF FMRI IN NEURODEGENERATIVE DISEASES}

Functional MRI has been applied in a number of ways in studies of patients with neurodegenerative diseases, and many of these applications are illustrative of how this technology can be applied to other neurological disorders.

Given the growing body of evidence that alterations in synaptic function are present very early in the pathophysiological process of $\mathrm{AD}$ and related disorders, possibly long before the development of clinical symptoms and even significant neuropathology, ${ }^{10-12}$ fMRI may be particularly useful in detecting alterations in brain function that may be present very early in the course of $\mathrm{AD}$ or related dementias. The fMRI is already beginning to reveal novel insights into functional abnormalities in particular brain regions in MCI, which in many cases is the earliest clinical phase of $\mathrm{AD}$ prior to dementia.

\section{Patterns of abnormal regional brain activation during task performance in $\mathrm{AD}$ and $\mathrm{MCI}$}

Functional MRI has been used to investigate abnormalities in patterns of regional brain activation during a variety of cognitive tasks in patients diagnosed with mild AD compared to control subjects. It is important to keep in mind that the particular abnormalities found in an fMRI study of an AD or other patient group are heavily dependent on the type of behavioral task used in the study; if the task does not engage a particular circuit, functional abnormalities will not likely be observed (see FIG. 1). Moreover, even brain regions not usually thought to be affected by AD (sensorimotor areas) have been shown to exhibit abnormal function in $\mathrm{AD}$ patients. ${ }^{13,14}$

In addition to memory, aspects of language and attention have been studied in AD patients using fMRI. Altered patterns of frontal and temporal activation have been observed in $\mathrm{AD}$ patients performing language tasks. ${ }^{15-17}$ Similarly, although temporoparietal activation was found to be diminished in AD during performance of semantic memory task, increased activation in temporal and frontal regions was also observed, suggesting possible compensatory processes. ${ }^{18}$ During performance of a visual attention task, $\mathrm{AD}$ patients were found to have abnormal parietal activation; increased prefrontal activation was also observed compared with controls, again suggesting possible compensatory mechanisms. ${ }^{19}$

With respect to memory, a number of fMRI studies in patients with clinically diagnosed $\mathrm{AD}$, using a variety of visual stimuli, have identified decreased activation in hippocampal and para-hippocampal regions compared to control subjects during episodic encoding tasks. ${ }^{20-25}$ Neocortical abnormalities in AD have also been demonstrated using fMRI, including decreased activation in temporal and prefrontal regions. ${ }^{22}$ In addition to $\mathrm{AD}$ related differences in task-related blood-oxygen level dependent (BOLD) signal amplitude or spatial extent, the temporal dynamics of activation appear to be altered in patients with $\mathrm{AD} .^{26}$ Interestingly, as has been observed in other types of tasks, increased activation in prefrontal 


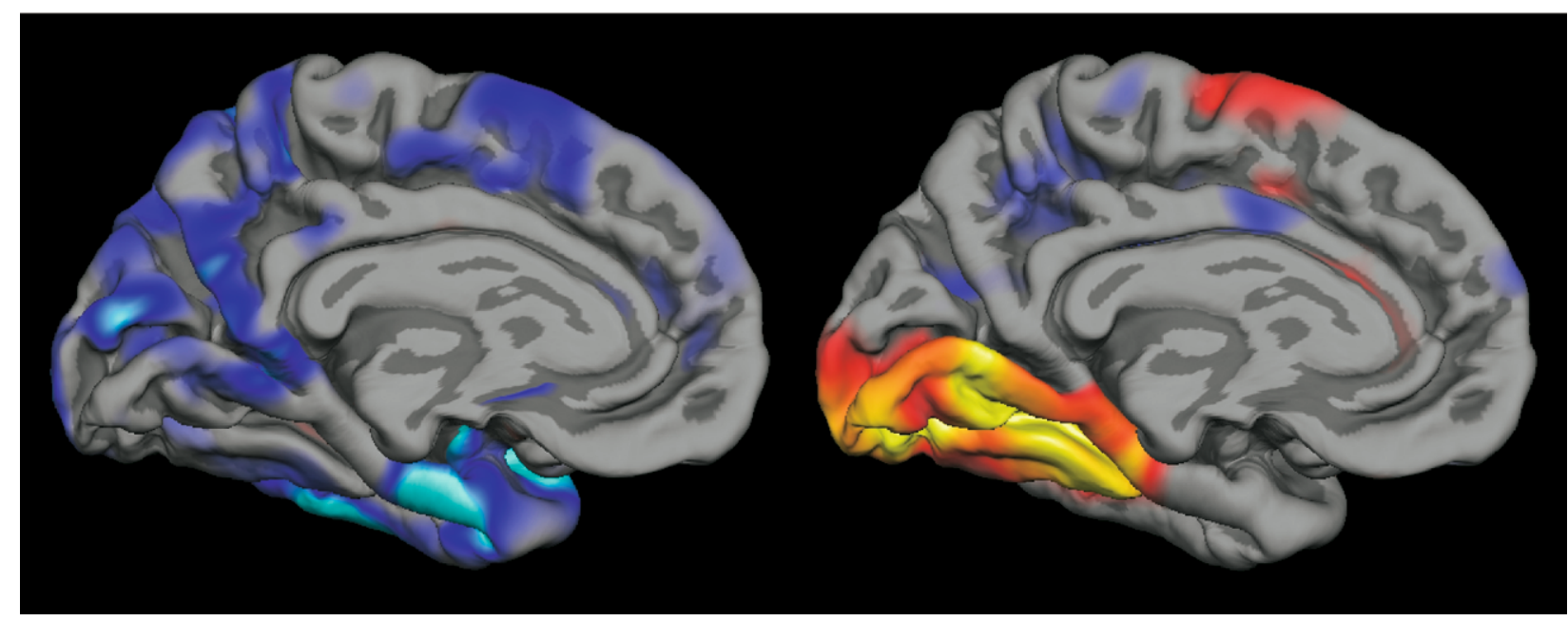

FIG. 1. The localization, magnitude, and extent of abnormalities observed in functional magnetic resonance imaging (fMRI) studies of patients with neurological diseases depend on both localization and severity of pathology and on functional networks engaged by the particular fMRI task, as well as participant performance on the task. In this illustration, regions of cortical thinning in Alzheimer's disease from structural MRI (left ${ }^{106}$ ) are compared with cortical areas activated, as measured with fMRI, in normals during an event-related study of successful learning of new information that was later able to be freely recalled $\left(\right.$ right $^{107}$ ).

and other regions has also been found in $\mathrm{AD}$ patients performing memory tasks. ${ }^{23}$

Whereas memory task-related fMRI data regarding medial temporal lobe (MTL) activation in individuals with MCI are less consistent than data from patients diagnosed with $\mathrm{AD}$, with reports of both decreased and increased activation, ${ }^{24,20,27}$ they do indicate that differences are present in comparison to older controls. Some of the variability in fMRI data in MTL activation appears to relate to degree of impairment along the spectrum of MCI, which suggests that fMRI may be sensitive to relatively subtle clinical differences in disease severity. ${ }^{27}$

\section{Compensatory hyperactivation: a universal adaptation response to brain injury?}

Recent data suggest that there is a phase of increased MTL activation in MCI (see FIG. 2) that may represent an attempted compensatory response to $\mathrm{AD}$ neuropathology, given that greater activation is associated with better memory performance, as well as smaller hippocampal volume..$^{25,27-29}$ The use of event-related fMRI paradigms $^{30}$ may help determine whether increased activation in MCI patients is specifically associated with successful memory, as opposed to a general effect that is present regardless of success (possibly indicating increased effort). For example, the results of a recent fMRI study using an event-related paradigm suggest that MTL hyperactivation in MCI is specifically seen during the encoding of new material that is later successfully recognized, supporting the compensatory hypothesis. ${ }^{31}$

Evidence is accumulating that task-related regional brain hyperactivation occurs in a variety of neuropsychiatric disorders and conditions, including AD/MCI, Huntington's disease, ${ }^{32}$ Parkinson's disease,${ }^{33}$ cerebrovascular disease, ${ }^{34,35}$ multiple sclerosis, ${ }^{36-38}$ traumatic brain injury, ${ }^{39}$ human immunodeficiency virus (HIV),${ }^{40}$ alcoholism, ${ }^{41}$ schizophrenia, ${ }^{42}$ sleep deprivation, ${ }^{43}$ and aging. ${ }^{44}$ All of these studies provide behavioral evidence that greater activation in task-relevant brain regions

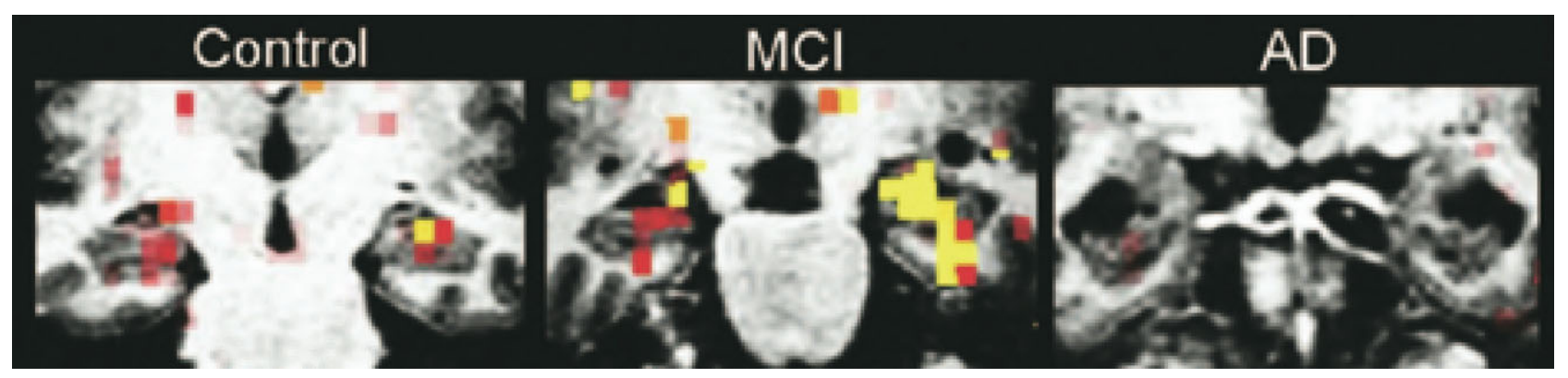

FIG. 2. A phase of compensatory hyperactivation appears to occur in the medial temporal lobe (MTL) in mild cognitive impairment, prior to the clinical onset of Alzheimer's disease (AD) dementia. Representative single subjects from each group, showing normal memoryrelated MTL activation measured with functional magnetic resonance imaging in normal older controls (left), hyperactivation and very mild atrophy in mild cognitive impairment (MCl) (middle), and hypoactivation and more prominent atrophy in mild $A D$ (right). ${ }^{28}$ Figure modified from published version. 
serves, at least in part, to preserve task performance in the patient group, supporting the contention that hyperactivation may be compensatory for neurological insults. It is possible, however, that hyperactivation reflects inefficient function of neural circuits in the face of injury, and that such a response may be deleterious in the long run. Thus, it will be critical to elucidate the relationships between behavioral performance, neural circuit function, and clinical course of disease, with the ultimate goal of determining how best to use these fMRI measures as biomarkers of putative therapeutic response in clinical trials.

\section{The modulatory effects of genetic risk factors for neurological disease on brain activation}

In the last few years there has been an explosion of literature on imaging and genetics, primarily in psychiatric disorders ${ }^{45}$ and regarding the basic science of genetic modulators of brain function. ${ }^{46}$ This is an area that is ripe for study in neurological disease, with a number of studies having been done in populations at elevated genetic risk for $\mathrm{AD}$.

The $A P O E \in 4$ allele is a major genetic susceptibility factor associated with increased risk for AD. ${ }^{47}$ Several fMRI studies have investigated regional brain activation during task performance in cognitively intact subjects stratified by their $A P O E$ allele status. Smith et al. ${ }^{48}$ reported decreased activation in inferior temporal regions with a visual naming and a letter fluency fMRI paradigm (there was no hippocampal or other MTL activation reported with these tasks) in $A P O E \in 4$ carriers. In a subsequent report, this group reported increased parietal activation in women with an APOE $\epsilon 4$ allele. $^{49}$ Bookheimer et al. ${ }^{50}$ reported increased activation in left hippocampal, parietal, and prefrontal regions among APOE $\epsilon 4$ carriers, compared to noncarriers, using a word-pair associative memory paradigm. In addition, an increased number of activated regions in the left hemisphere at baseline was associated with a decline in memory at the 2-year follow-up among the APOE $\epsilon 4$ carriers. The authors hypothesized that this increase in activation in the $A P O E \in 4$ carriers might represent the additional cognitive effort or neuronal recruitment required to adequately perform the task. Similarly increased activation in multiple brain regions was recently reported in cognitively intact $A P O E \epsilon 4$ carriers compared to $\epsilon 3$ carriers, although the effect was lateralized to the right MTL region (left hippocampal activation was greater in $\epsilon 3$ carriers). ${ }^{51}$ Among a group of 29 controls, MCI subjects, and $\mathrm{AD}$ patients, we recently reported that 13 APOE $\epsilon 4$ carriers demonstrated greater entorhinal activation than noncarriers, in the absence of genotype-related differences in the volumes of these regions. ${ }^{28}$ Other studies suggest that decreased MTL activation may also be seen in APOE $\epsilon 4$ carriers. ${ }^{52}$

\section{fMRI in the differential diagnosis of neuropsychiatric syndromes}

The early detection and differential diagnosis of disorders causing cognitive impairment is a promising aim for further work using fMRI. Because clinical evaluation and neuropsychological testing are currently the most sensitive approaches to diagnosis, and fMRI is sensitive to both cognitive performance and clinical status, it seems reasonable to hope that the potential capability of fMRI to detect alterations in the pattern and degree of regional brain activation during task performance may provide additional useful data to complement clinical and psychometric evaluations. However, relatively little fMRI data have been published on differential diagnosis to date.

In elderly individuals with cognitive symptoms, it can be difficult to distinguish a neurodegenerative process from depression. Functional MRI may be helpful in this setting. In a study of individuals who had sought clinical evaluation for memory-related symptoms, Gron et al. ${ }^{53}$ investigated the utility of fMRI to differentiate patterns of regional brain activation in those diagnosed with depression versus $\mathrm{AD}$ (as well as a control group). Hippocampal activation during the memory task was decreased in $\mathrm{AD}$ patients compared with controls and depressed patients. In contrast, orbitofrontal and cingulate activation were greater in depressed patients than in AD subjects and controls.

Furthermore, different forms of neurodegenerative dementias may be challenging to specifically diagnose early in their course. Functional MRI may provide helpful data to assist in differential diagnosis of the dementias. Rombouts et al. ${ }^{54}$ compared regional brain activation during a working memory task in patients with frontotemporal dementia to that of $\mathrm{AD}$ patients. Although both groups activated similar fronto-parietal -thalamic regions, fronto-parietal activation was diminished in frontotemporal dementia patients than in AD patients.

Further insights into the utility of fMRI in assisting with differential diagnosis may be potentially gained through prospective studies of patients presenting for clinical evaluation with subtle symptoms consistent with a degenerative dementia who do not yet have a clear clinical diagnosis. If such individuals are scanned using tasks they can still perform and then be clinically followed, it may be possible to determine whether fMRI has predictive power in differential diagnosis.

\section{fMRI as a predictive biomarker}

We recently pursued such a study of a group of 25 senior citizens spanning the spectrum of MCI, none of whom were demented at the time of baseline assessment, but who exhibited varying degrees of mild symptoms of cognitive impairment clinically (as measured using the clinical demensia rating (CDR) Sum-of-Boxes (CDR- 


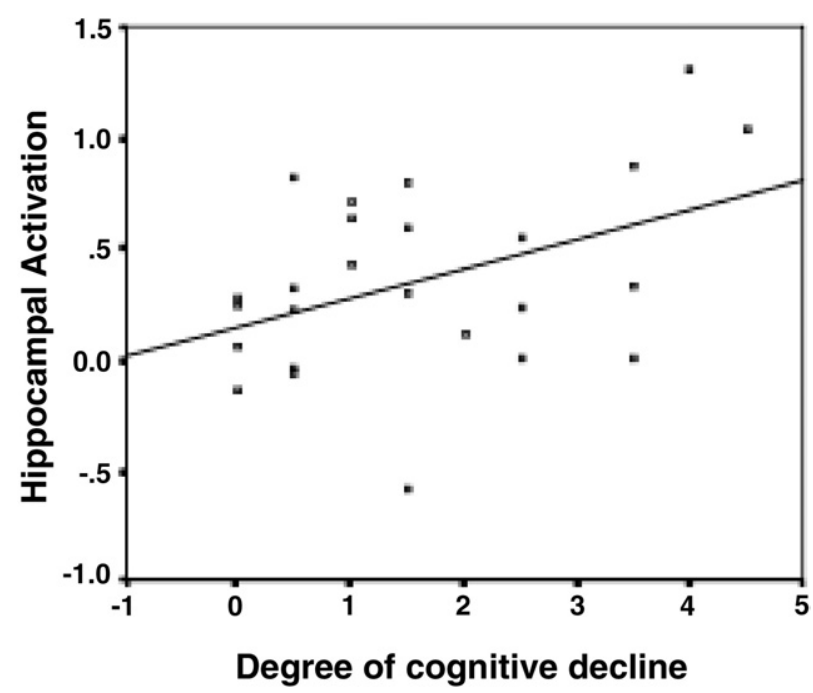

FIG. 3. Functional magnetic resonance imaging (fMRI) as a predictive quantitative imaging biomarker. In a group of mild cognitive impairment patients, hippocampal activation at baseline predicts the degree of cognitive decline during 4-year timeframe after scanning. ${ }^{55}$ The $\mathrm{Y}$ axis indicates percent blood-oxygen level dependent (BOLD) signal change within hippocampal formation; $\mathrm{X}$ axis indicates relative clinical decline as measured by CDR Sum-of-Boxes (CDR-SB) scale, with higher numbers indicating greater decline.

SB) scale. ${ }^{55}$ At baseline, subjects performed a visual scene-encoding task during fMRI scanning and were clinically followed longitudinally after scanning. During $\sim 4$ years of follow-up after scanning, subjects demonstrated a wide range of cognitive decline, with some showing no change and others progressing to dementia (i.e., a change in CDR-SB that ranged from 0 to 4.5). The degree of cognitive decline was predicted by hippocampal activation at the time of baseline scanning, with greater hippocampal activation predicting greater decline $(p<0.05)$ (see FIG. 3). This finding was present even after controlling for baseline degree of impairment (CDR-SB), age, education, and hippocampal volume. These data suggest that fMRI may provide a physiological imaging biomarker useful for identifying the subgroup of MCI individuals at highest risk of cognitive decline for potential inclusion in disease-modifying clinical trials.

\section{Links between task-related brain function abnormalities and altered resting brain activity}

Recent fMRI studies are beginning to reveal a link between disease-related hemodynamic alterations and the well-described resting perfusion/metabolic abnormalities in AD. Hypoperfusion/metabolism is typically seen with nuclear medical imaging techniques (such as FDG-PET or single photon emission computed tomography) in temporo-parietal/posterior cingulate cortical regions in $\mathrm{AD}$ patients during the "resting" state. The medial parietal/posterior cingulate cortex, along with medial frontal and lateral parietal regions, appear to com- pose a "default mode" network that is more active when individuals are not engaged in particular tasks, and which is thought to play a role in vigilance, readiness, or monitoring - these regions "deactivate" (BOLD signal amplitude falls below baseline) during cognitive task performance. ${ }^{56}$ Several recent studies in AD patients have demonstrated alterations in the deactivation and functional connectivity of these regions, suggesting that this default mode network is disrupted by the disease. ${ }^{25,57-59}$ Substantial overlap is present between these default mode areas and the localization of PET amyloid tracer binding. ${ }^{60}$

\section{fMRI STUDIES OF BRAIN PLASTICITY}

A growing body of fMRI literature has emerged that directly demonstrates correlates of regional brain plasticity in neurological disorders. Much of this work has been performed in patients who have suffered focal ischemic strokes. ${ }^{61,62}$ This population is attractive because of the relatively rapid pace of functional recovery in many cases, such that studies can be performed during a period of months, minimizing the other variables that may potentially contribute to changes in fMRI signal in longitudinal studies (e.g., patient-related variables in those with progressive diseases; instrument-related variables resulting from scanner upgrades).

Functional MRI studies of stroke patients have shown that, for example, the laterality of primary sensorimotor cortex activation may shift after an infarct from typical contralateral organization (which would be ipsilesional during movement of the body part affected by the stroke) toward the side ipsilateral to movement. Furthermore, the manner by which a behavior is normally organized influences how that behavior is organized after a stroke. Thus, for example, face motor representation (i.e., normally bilaterally organized at a neural level) is more likely to be shifted to the hemisphere ipsilateral to movement than a movement that is less bilaterally organized in the normal state (see FIG. 4).

The ready availability of instruments for fMRI studies, their safety in longitudinal studies, and the ability to combine fMRI measures with other brain mapping tools has contributed to the explosion of plasticity research in stroke and other neurological disorders. This will likely be an area in which findings from basic imaging studies are translated quickly into assessment tools and imaging biomarkers useful for prognostication. ${ }^{63}$

\section{SINGLE CASE $(N=1)$ fMRI STUDIES}

The grand tradition in neurology of single case reports has entered a modern era in which brain physiological mechanisms can be investigated with relative ease because many clinical MRI systems are being equipped for 


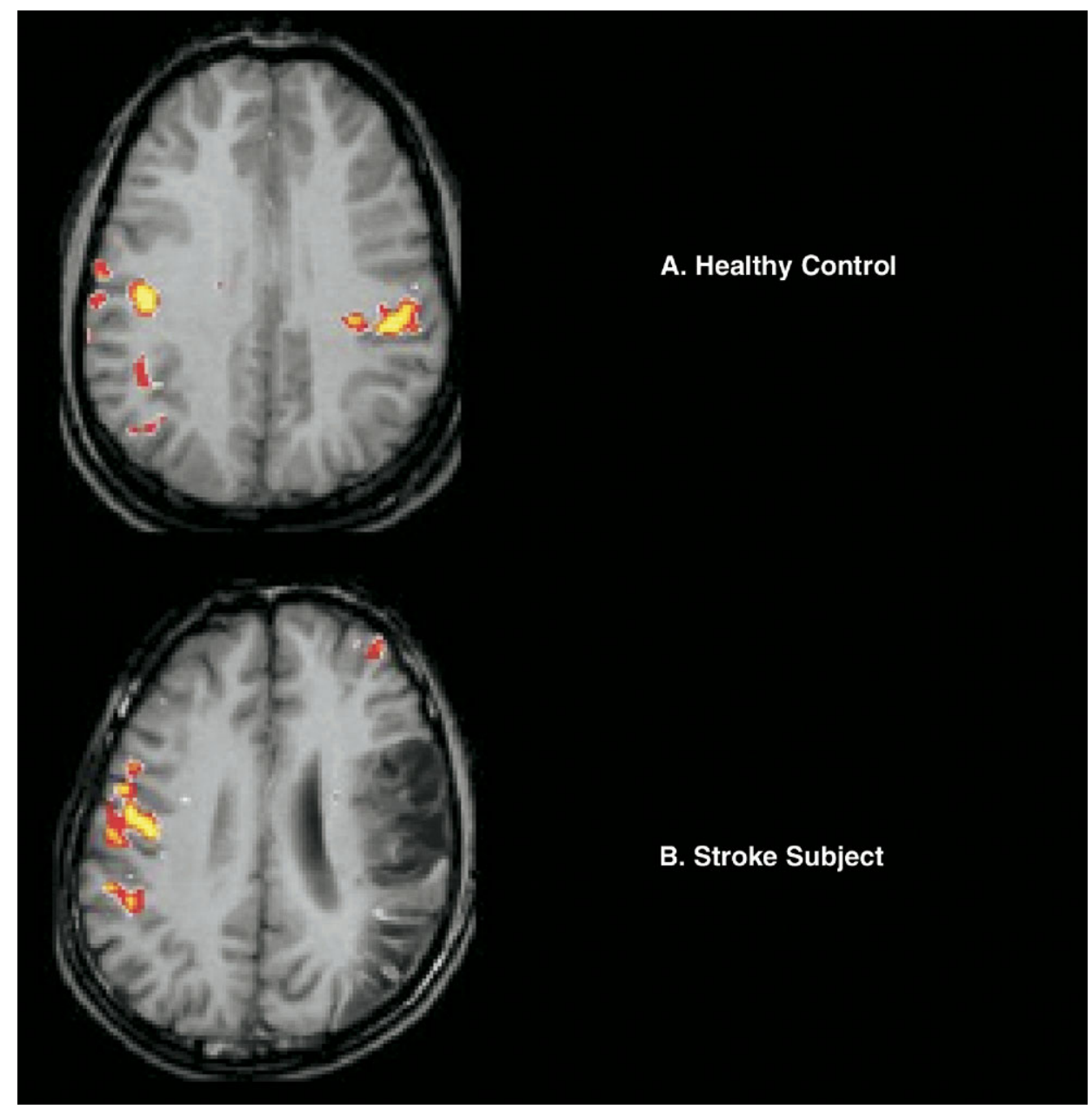

FIG. 4. Functional magnetic resonance imaging (fMRI) measurements of regional brain activation during movement of the right face. In the normal subject (A, Healthy Control, top), face movement is bilaterally organized. In the subject with a good chronic clinical outcome after stroke (B, Stroke Subject, bottom), movement arises from only the contralesional side. Image courtesy of Dr. Steve Cramer. ${ }^{109}$

fMRI capability. Single case fMRI studies have begun to illuminate the neural correlates of a variety of neurological disorders. A woman's poststroke phantom limb sensations were shown to relate to medial prefrontal activity near the supplementary motor area. ${ }^{64}$ In a young man with developmental amnesia due to perinatal hypoxemia, despite 50\% reduction in hippocampal volume compared to controls, retrieval of real-world memories was associated with bilateral hippocampal activation. ${ }^{65}$ Motor recovery in a patient who underwent multiple subpial transection of the primary motor cortex for a seizure disorder was initially associated with expanded bilateral motor system recruitment, followed later by return to presurgical motor organization within the transected cortex. ${ }^{66}$

Five members of a family with familial AD were studied using fMRI-monitored memory tasks; in this family, an autosomal-dominant presenilin mutation leads to $\mathrm{AD}$, typically by 48 years of age. ${ }^{67}$ In a young $(20-$ year-old) mutation carrier, increased hippocampal and frontotemporal activation was observed during an epi- sodic-memory task, whereas decreased activation in these regions was found in a middle-aged (45-year-old) mutation carrier, compared with activation in the three family members without the mutation (i.e., one middleaged and two young adults) and with a group of young, unrelated controls (mean age, 22 years of age). The authors infer that these findings are consistent with the early hyperactivation model of predementia functional brain change in $\mathrm{AD}$, as previously described.

A young woman in a persistent vegetative state was shown to exhibit differential regional brain activity when given two sets of verbal instructions, to which she showed no behavioral response. ${ }^{68}$ Supplementary motor activity was present when she was asked to "imagine playing tennis," whereas posterior parietal and para-hippocampal activity was present when she was asked to "imagine visiting the rooms in your home." Although the clinical implications of these data are far from clear, it is a striking example of how imaging technologies can shed light on the level of 
brain function of which a patient may be capable, even when behavior is not revealing.

Although the growing number of examples of fMRI single case studies suggest that this and related functional neuroimaging tools may have great potential for clinical use, challenges in the interpretation of these $(n=$ 1) imaging data emphasize the need for rigorous clinical behavioral methodology at the same time.

\section{PHARMACOLOGIC FMRI}

Pharmacological fMRI (phMRI) is being increasingly used to study the effects of neurochemical perturbations on brain function at a regionally-specific or systems level. ${ }^{69,70}$ Besides the cholinergic system, phMRI has been applied to studies of dopaminergic modulation of working memory, ${ }^{71}$ gamma amino butyric acid (GABA)ergic modulation of repetition priming, ${ }^{72}$ and noradrenergic effects on attention/orienting tasks. ${ }^{73}$ In addition, fMRI has been used to study the effects of intrinsic or extrinsic steroid hormonal modulation on cognitive tasks. $^{74,75}$ The PhMRI has begun to reveal regional brain activation responses associated with individual differences in behavioral response to pharmacological agents. ${ }^{76} \mathrm{In}$ clinical populations, phMRI has been applied to investigations of cocaine dependence, ${ }^{77}$ depression, ${ }^{78}$ schizophrenia, ${ }^{79}$ and Parkinson's disease. ${ }^{80}$ A recently emerging, exciting application of phMRI is in the prediction of clinical response to pharmacological treatment. For example, patients with depression who are treated with antidepressant medications demonstrate changes in activation of affective circuitry that are associated with clinical response to pharmacological treatment, ${ }^{81}$ which may predict treatment response. ${ }^{82}$ The addition of genetic factors to these investigations holds fascinating and powerful promise to illuminate fundamental pharmacogenetic mechanisms of CNS drug effects. For example, in schizophrenic patients, there is a catechol-o-methyl-transferase polymorphism-dependent effect of atypical antipsychotic therapy on prefrontal activation during working memory task performance. ${ }^{83}$

With respect to the cholinergic system, phMRI has been used to study the effects of cholinergic antagonists or agonists on brain function and behavior in healthy young individuals ${ }^{9,84-87,72,88}$ and on aging. ${ }^{89}$ Initial studies of cholinergic enhancement in AD showed increased fusiform activation during episodic encoding ${ }^{90}$ and cholinergic inhibition in controls was associated with reduced fusiform, hippocampal, and prefrontal activation during episodic encoding. ${ }^{9}$ Several subsequent studies have used fMRI to identify modulatory effects of cholinergic agents in patients with MCI. ${ }^{91-93}$

Although findings from studies in a variety of subject populations demonstrate the utility of phMRI as an important investigative tool, several methodological issues need to be considered when data are being interpreted. ${ }^{94}$ In attempting to measure specific pharmacological effects on regional neural activity, phMRI may be confounded by global or nonspecific actions. For example, a drug may induce systemic changes in blood flow or alterations in neurovascular coupling, and these effects may differ between the two patient populations being compared (i.e., AD patients and controls). For example, there appears to be regional differences in the BOLD signal response properties in $\mathrm{AD}$ patients compared to controls. ${ }^{13,14}$ It has also been argued that cholinergic drugs may affect neurovascular coupling, ${ }^{95}$ although imaging studies to date have not demonstrated global pharmacological effects; sensory and motor areas are spared by cholinergic inhibition. ${ }^{9,72}$ Many phMRI studies seek to identify drug effects that show differential task-condition specificity and have begun to identify such effects in studies of human memory function that relate to similar known neurophysiological effects in animals. ${ }^{96}$

\section{TECHNICAL ADVANCES IN HIGH- RESOLUTION AMRI}

A number of technical advances in fMRI are emerging that will enable investigators to generate and test new hypotheses regarding brain function and dysfunction in neurological disorders. Among others, these advances include improved data acquisition techniques, data analysis techniques, and the integration of fMRI with other measures, such as structural MRI, ${ }^{27,97}$ diffusion tensor imaging, ${ }^{63}$ PET of ligands for metabolism (e.g., fleurodeoxyglucose), neurotransmitter systems (e.g., cholinergic or dopaminergic tracers), or pathological markers (e.g., PIB or FDDNP AD pathology tracers), electroencephalography, ${ }^{98,99}$ and transcranial magnetic stimulation. ${ }^{100}$ For the purposes of this review, we will focus on advances in data acquisition techniques that aim to improve the spatial resolution of fMRI data.

Besides enabling hypothesis testing regarding fine functional organization of particular brain regions, advantages of higher resolution fMRI data include a better capacity to visualize anatomical features directly from functional data, and thus confirm localization of activation and improved accuracy of co-registration of functional data with structural data. These improvements should result in better sensitivity to functionally specific brain subregions, and better specificity with respect to functional abnormalities in these subregions in the early or mild phases of neuropsychiatric illness.

Using current generation, widely available instrumentation, it is possible to obtain whole-brain coverage in most types of fMRI experiments at $\sim 3.5$ to $4 \mathrm{~mm}$ or slightly higher resolution. The major tradeoffs for obtaining fMRI data at a higher resolution include partial brain coverage due to memory limitations of current 


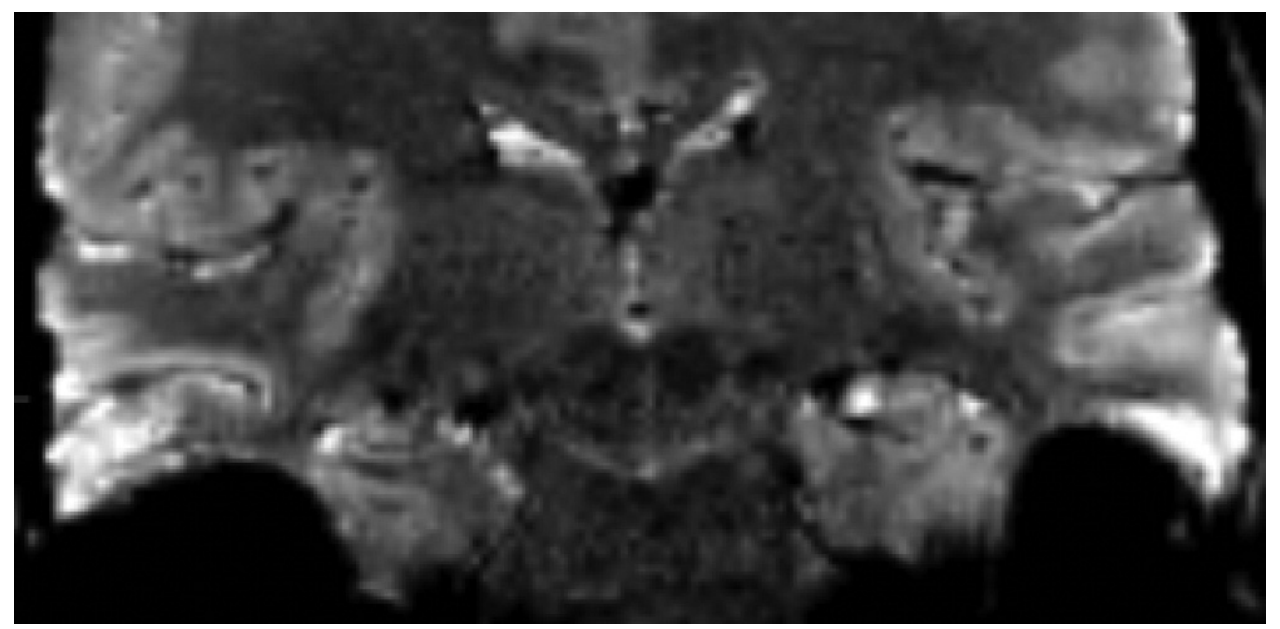

FIG. 5. The 1-mm isotropic echo planar functional magnetic resonance imaging (fMRI) data obtained using 32-channel head coil on a 3.0 Tesla system. Acceleration factor $=2$ was used to reduce susceptibility artifact. Data obtained by Dickerson BC, Wiggins GC, Benner T, Wald LL, Massachusetts General Hospital, Boston, MA.

scanner systems, slower repetition times, signal-to-noise reduction (SNR), and BOLD contrast-to-noise reduction (CNR), the signal property that enables functional activation to be detected.

\section{Parallel imaging with large-N array head coils}

Advances are being made in phased array head coil technology that enable parallel imaging with an increasing number of receiver elements (see review article by Katscher et al. ${ }^{101}$ ). For example, 12- to 16-channel head coils are becoming readily available for use with most contemporary scanning systems. The use of parallel imaging acceleration factors can reduce susceptibility artifacts in areas of the brain such as the MTL, but at a cost of reduced SNR and BOLD CNR. ${ }^{102}$ Through their boost in signal, larger array head coils can help compensate for this reduction in SNR/CNR. Custom-built receiver head coils with 32 or more channels are now in development at research sites and have demonstrably improved SNR, even in deep brain regions. ${ }^{103}$ We recently used a 32channel coil (built by Drs. G. Wiggins and L. Wald at
Massachusetts General Hospital) at $3.0 \mathrm{~T}$ to obtain $1 \mathrm{~mm}$ isotropic fMRI data with an echo-planar sequence (see FIG. 5). ${ }^{104}$

\section{Ultra-high field imaging}

The use of ultra-high field (e.g., 7.0 or 9.4 Tesla) MRI systems will also likely lead to the capability to obtain higher-resolution fMRI data with improved BOLD contrast over lower field systems. Although a few of these systems worldwide are beginning to yield results in human studies, ${ }^{105}$ there are a host of technical challenges that need to be surmounted for fMRI studies, including the larger susceptibility, blood vessel, and other artifacts present at ultra-high field. New fMRI sequences are being developed that are less sensitive to susceptibility artifacts and other sources of signal dropout. ${ }^{106}$ We recently used a stack-of-segmented EPI sequence on our 7.0 Tesla system to obtain minimally distorted data at 1 $\mathrm{mm}$ isotropic using a simple visual cortex activation task (see FIG. 6). ${ }^{104}$
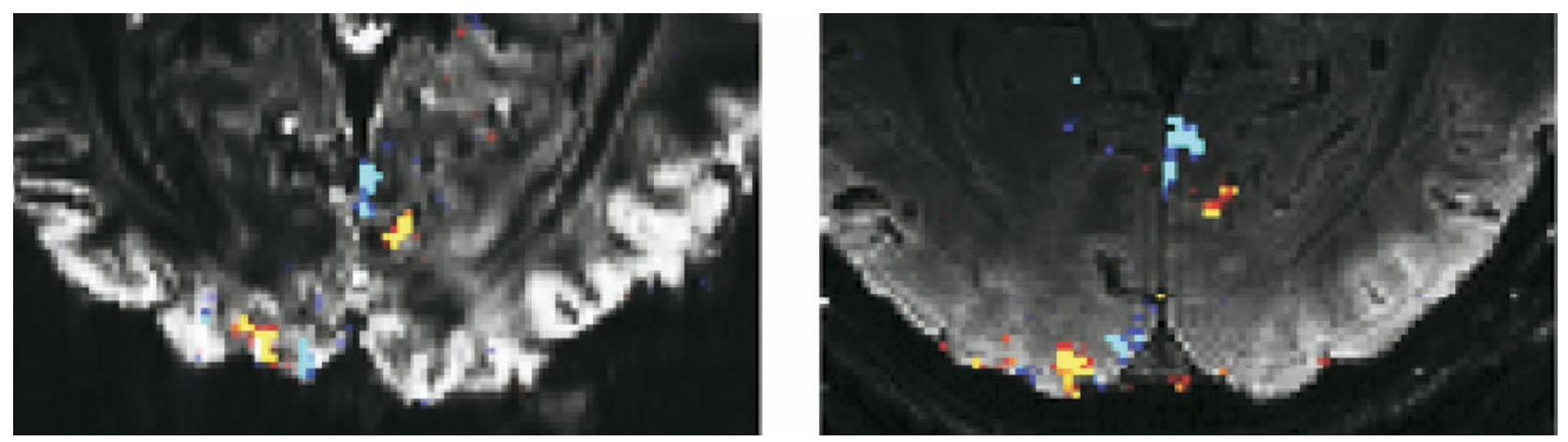

FIG. 6. Ultra-high field (7.0 Tesla) functional magnetic resonance imaging (fMRI) of visual cortex activation with 1-mm isotropic voxels using 2-dimensional echo-planar sequence (left) versus 3-dimensional stack-of-segmented echo-planar sequence (right), illustrating similar functional topography to differentially-oriented wedge-shaped flickering checkerboards (blue versus red/yellow) with notable reduction in distortion. ${ }^{103}$ 


\section{CONCLUSIONS}

Functional MRI is a particularly attractive method for use by clinical investigators to study task-related brain activation in patients with neurological or neuropsychiatric illness. Despite the relative infancy of the field, there have already been a number of promising fMRI studies in neurodegenerative, demyelinating, cerebrovascular, and other neurological disorders that highlight the potential uses of fMRI in both basic and clinical spheres of investigation. Functional MRI may provide novel insights into the neural correlates of cognitive and other abilities, ${ }^{107}$ and how they are altered by neurological disease and medications. ${ }^{108}$ The technique may help elucidate fundamental aspects of brain-behavior relationships, such as the genetic influences on task-related brain physiology. Functional MRI measures hold promise for multiple clinical applications, including the early detection and differential diagnosis, predicting future change in clinical status, and as a marker of alterations in brain physiology related to neurotherapeutic agents. The greatest potential of fMRI likely lies in the study of very early and preclinical stages of progressive neurological diseases at the point of subtle neuronal dysfunction, prior to overt anatomic pathology. ${ }^{109}$ There is a need for further validation and reliability studies and continued technical advances to fully realize the potential of fMRI.

Acknowledgments: This work was supported by the National Institutes of Health grant (NIH K23-AG22509). The high-resolution functional MRI work described was also supported by the NCRR (P41-RR14075), the Mental Illness and Neuroscience Discovery (MIND) Institute, and the Athinoula A. Martinos Center for Biomedical Imaging.

\section{REFERENCES}

1. Just MA, Cherkassky VL, Keller TA, Kana RK, Minshew NJ. Functional and anatomical cortical underconnectivity in autism: evidence from an FMRI study of an executive function task and corpus callosum morphometry. Cereb Cortex 2007;17:951-961.

2. Holt DJ, Kunkel L, Weiss AP, et al. Increased medial temporal lobe activation during the passive viewing of emotional and neutral facial expressions in schizophrenia. Schizophr Res 2006;82: $153-162$.

3. Thermenos HW, Seidman LJ, Poldrack RA, et al. Elaborative verbal encoding and altered anterior parahippocampal activation in adolescents and young adults at genetic risk for schizophrenia using FMRI. Biol Psychiatry 2007;61:564-574.

4. Dickerson BC, Sperling RA. Neuroimaging biomarkers for clinical trials of disease-modifying therapies in Alzheimer's disease. Neurorx 2005;2:348-360.

5. Burock MA, Buckner RL, Woldorff MG, Rosen BR, Dale AM. Randomized event-related experimental designs allow for extremely rapid presentation rates using functional MRI. Neuroreport 1998;9:3735-3739.

6. Price CJ, Friston KJ. Scanning patients with tasks they can perform. Hum Brain Mapp 1999;8:102-108.

7. Machielsen WC, Rombouts SA, Barkhof F, Scheltens P, Witter MP. FMRI of visual encoding: reproducibility of activation. Hum Brain Mapp 2000;9:156-164.

8. Manoach DS, Halpern EF, Kramer TS, et al. Test-retest reliability of a functional MRI working memory paradigm in normal and schizophrenic subjects. Am J Psychiatry 2001;158:955-958.
9. Sperling R, Greve D, Dale A, et al. Functional MRI detection of pharmacologically induced memory impairment. Proc Natl Acad Sci U S A 2002;99:455-460.

10. Scheff SW, Price DA, Schmitt FA, Mufson EJ. Hippocampal synaptic loss in early Alzheimer's disease and mild cognitive impairment. Neurobiol Aging 2006;27:1372-1384.

11. Selkoe DJ. Alzheimer's disease is a synaptic failure. Science 2002;298:789-791.

12. Coleman P, Federoff H, Kurlan R. A focus on the synapse for neuroprotection in Alzheimer disease and other dementias. Neurology 2004;63:1155-1162.

13. Buckner RL, Snyder AZ, Sanders AL, Raichle ME, Morris JC. Functional brain imaging of young, nondemented, and demented older adults. J Cogn Neurosci 2000;12(suppl 2):24-34.

14. D'Esposito M, Deouell LY, Gazzaley A. Alterations in the BOLD fMRI signal with ageing and disease: a challenge for neuroimaging. Nat Rev Neurosci 2003;4:863-872.

15. Grossman M, Koenig P, DeVita C, et al. Neural basis for verb processing in Alzheimer's disease: an fMRI study. Neuropsychology 2003;17:658-674.

16. Johnson SC, Saykin AJ, Baxter LC, et al. The relationship between fMRI activation and cerebral atrophy: comparison of normal aging and Alzheimer disease. Neuroimage 2000;11:179-187.

17. Saykin AJ, Flashman LA, Frutiger SA, et al. Neuroanatomic substrates of semantic memory impairment in Alzheimer's disease: patterns of functional MRI activation. J Int Neuropsychol Soc 1999;5:377-392.

18. Grossman M, Koenig P, Glosser G, et al. Neural basis for semantic memory difficulty in Alzheimer's disease: an fMRI study. Brain 2003;126:292-311.

19. Thulborn KR, Martin C, Voyvodic JT. Functional MR imaging using a visually guided saccade paradigm for comparing activation patterns in patients with probable Alzheimer's disease and in cognitively able elderly volunteers. AJNR Am J Neuroradiol 2000;21:524-531.

20. Small SA, Perera GM, DeLaPaz R, Mayeux R, Stern Y. Differential regional dysfunction of the hippocampal formation among elderly with memory decline and Alzheimer's disease. Ann Neurol 1999;45:466-472.

21. Rombouts SA, Barkhof F, Veltman DJ, et al. Functional MR imaging in Alzheimer's disease during memory encoding. AJNR Am J Neuroradiol 2000;21:1869-1875.

22. Kato T, Knopman D, Liu H. Dissociation of regional activation in mild AD during visual encoding: a functional MRI study. Neurology 2001;57:812-816.

23. Sperling RA, Bates JF, Chua EF, et al. fMRI studies of associative encoding in young and elderly controls and mild Alzheimer's disease. J Neurol Neurosurg Psychiatry 2003;74:44-50.

24. Machulda MM, Ward HA, Borowski B, et al. Comparison of memory fMRI response among normal, MCI, and Alzheimer's patients. Neurology 2003;61:500-506.

25. Celone KA, Calhoun VD, Dickerson BC, et al. Alterations in memory networks in mild cognitive impairment and Alzheimer's disease: an independent component analysis. J Neurosci 2006;26: 10222-10231.

26. Rombouts SARB, Goekoop R, Stam CJ, Barkhof F, Scheltens P. Delayed rather than decreased BOLD response as a marker for early Alzheimer's disease. Neuroimage 2005;26:1078-1085.

27. Dickerson BC, Salat DH, Bates JF, et al. Medial temporal lobe function and structure in mild cognitive impairment. Ann Neurol 2004;56:27-35.

28. Dickerson BC, Salat DH, Greve DN, et al. Increased hippocampal activation in mild cognitive impairment compared to normal aging and AD. Neurology 2005;65:404-411.

29. Hamalainen A, Pihlajamaki M, Tanila H, et al. Increased fMRI responses during encoding in mild cognitive impairment. Neurobiol Aging September 22, 2006 [Epub ahead of print].

30. Sperling R, Chua E, Cocchiarella A, et al. Putting names to faces: successful encoding of associative memories activates the anterior hippocampal formation. Neuroimage 2003;20:1400-1410.

31. Kircher T, Weis S, Freymann K, et al. Hippocampal activation in MCI patients is necessary for successful memory encoding. J Neurol Neurosurg Psychiatry February 7, 2007 [Epub ahead of print]. 
32. Rosas HD, Feigin AS, Hersch SM. Using advances in neuroimaging to detect, understand, and monitor disease progression in Huntington's disease. NeuroRx 2004;1:263-272.

33. Monchi O, Petrides M, Doyon J, Postuma RB, Worsley K, Dagher A. Neural bases of set-shifting deficits in Parkinson's disease. J Neurosci 2004;24:702-710.

34. Johansen-Berg H, Dawes H, Guy C, Smith SM, Wade DT, Matthews PM. Correlation between motor improvements and altered fMRI activity after rehabilitative therapy. Brain 2002;125:27312742.

35. Carey JR, Kimberley TJ, Lewis SM, et al. Analysis of fMRI and finger tracking training in subjects with chronic stroke. Brain 2002;125:773-788.

36. Reddy H, Narayanan S, Arnoutelis R, et al. Evidence for adaptive functional changes in the cerebral cortex with axonal injury from multiple sclerosis. Brain 2000;123:2314-2320.

37. Morgen K, Kadom N, Sawaki L, et al. Training-dependent plasticity in patients with multiple sclerosis. Brain 2004;127:25062517.

38. Buckle GJ. Functional magnetic resonance imaging and multiple sclerosis: the evidence for neuronal plasticity. J Neuroimaging 2005; 15:82S-93S.

39. McAllister TW, Saykin AJ, Flashman LA, et al. Brain activation during working memory 1 month after mild traumatic brain injury: a functional MRI study. Neurology 1999;53:1300-1308.

40. Ernst T, Chang L, Jovicich J, Ames N, Arnold S. Abnormal brain activation on functional MRI in cognitively asymptomatic HIV patients. Neurology 2002;59:1343-1349.

41. Desmond JE, Chen SH, DeRosa E, Pryor MR, Pfefferbaum A, Sullivan EV. Increased frontocerebellar activation in alcoholics during verbal working memory: an fMRI study. Neuroimage 2003;19:1510-1520.

42. Callicott JH, Mattay VS, Verchinski BA, Marenco S, Egan MF, Weinberger DR. Complexity of prefrontal cortical dysfunction in schizophrenia: more than up or down. Am J Psychiatry 2003;160: 2209-2215.

43. Drummond SP, Brown GG, Gillin JC, Stricker JL, Wong EC, Buxton RB. Altered brain response to verbal learning following sleep deprivation. Nature 2000;403:655-657.

44. Cabeza R, Anderson ND, Locantore JK, McIntosh AR. Aging gracefully: compensatory brain activity in high-performing older adults. Neuroimage 2002;17:1394-1402.

45. Winterer G, Hariri AR, Goldman D, Weinberger DR. Neuroimaging and human genetics. Int Rev Neurobiol 2005;67:325-383.

46. Hariri AR, Weinberger DR. Functional neuroimaging of genetic variation in serotonergic neurotransmission. Genes Brain Behav 2003;2:341-349.

47. Saunders AM. Apolipoprotein E and Alzheimer disease: an update on genetic and functional analyses. J Neuropathol Exp Neurol 2000;59:751-758.

48. Smith CD, Andersen AH, Kryscio RJ, et al. Altered brain activation in cognitively intact individuals at high risk for Alzheimer's disease. Neurology 1999;53:1391-1396.

49. Smith CD, Andersen AH, Kryscio RJ, et al. Women at risk for $\mathrm{AD}$ show increased parietal activation during a fluency task. Neurology 2002;58:1197-1202.

50. Bookheimer SY, Strojwas MH, Cohen MS, et al. Patterns of brain activation in people at risk for Alzheimer's disease. N Engl J Med 2000;343:450-456.

51. Bondi MW, Houston WS, Eyler LT, Brown GG. fMRI evidence of compensatory mechanisms in older adults at genetic risk for Alzheimer disease. Neurology 2005;64:501-508.

52. Johnson SC, Schmitz TW, Trivedi MA, et al. The influence of Alzheimer disease family history and apolipoprotein E $\epsilon 4$ on mesial temporal lobe activation. J Neurosci 2006;26:6069-6076.

53. Gron G, Bittner D, Schmitz B, Wunderlich AP, Riepe MW. Subjective memory complaints: objective neural markers in patients with Alzheimer's disease and major depressive disorder. Ann Neurol 2002;51:491-498.

54. Rombouts SA, van Swieten JC, Pijnenburg YA, Goekoop R, Barkhof F, Scheltens P. Loss of frontal fMRI activation in early frontotemporal dementia compared to early AD. Neurology 2003; 60:1904-1908.
55. Miller S, Bates J, Blacker D, Sperling RA, Dickerson BC. Hippocampal activation in MCI predicts subsequent cognitive decline. Presented at the International Conference on Alzheimer's Disease; July 17, 2006; Madrid.

56. Raichle ME, MacLeod AM, Snyder AZ, Powers WJ, Gusnard DA, Shulman GL. A default mode of brain function. Proc Natl Acad Sci U S A 2001;98:676-682.

57. Lustig C, Snyder AZ, Bhakta M, et al. Functional deactivations: change with age and dementia of the Alzheimer type. Proc Natl Acad Sci U S A 2003;100:14504-14509.

58. Greicius MD, Srivastava G, Reiss AL, Menon V. Default-mode network activity distinguishes Alzheimer's disease from healthy aging: evidence from functional MRI. Proc Natl Acad Sci U S A 2004;101:4637-4642.

59. Rombouts SARB, Barkhof F, Goekoop R, Stam CJ, Scheltens P. Altered resting state networks in mild cognitive impairment and mild Alzheimer's disease: an fMRI study. Hum Brain Mapp 2005;26:231-239.

60. Buckner RL, Snyder AZ, Shannon BJ, et al. Molecular, structural, and functional characterization of Alzheimer's disease: evidence for a relationship between default activity, amyloid, and memory. J Neurosci 2005;25:7709-7717.

61. Ward NS, Frackowiak RS. The functional anatomy of cerebral reorganization after focal brain injury. J Physiol Paris 2006;99: 425-436.

62. Hodics T, Cohen LG, Cramer SC. Functional imaging of intervention effects in stroke motor rehabilitation. Arch Phys Med Rehabil 2006;87:S36-S42.

63. Stinear CM, Barber PA, Smale PR, Coxon JP, Fleming MK, Byblow WD. Functional potential in chronic stroke patients depends on corticospinal tract integrity. Brain 2007;130:170-180.

64. McGonigle DJ, Hanninen R, Salenius S, Hari R, Frackowiak RS, Frith CD. Whose arm is it anyway? An fMRI case study of supernumerary phantom limb. Brain 2002;125:1265-1274.

65. Maguire EA, Vargha-Khadem F, Mishkin M. The effects of bilateral hippocampal damage on fMRI regional activations and interactions during memory retrieval. Brain 2001;124:11561170 .

66. Moo LR, Slotnick SD, Krauss G, Hart J. A prospective study of motor recovery following multiple subpial transections. Neuroreport 2002;13:665-669.

67. Mondadori CR, Buchmann A, Mustovic H, et al. Enhanced brain activity may precede the diagnosis of Alzheimer's disease by 30 years. Brain 2006;129:2908-2922.

68. Owen AM, Coleman MR, Boly M, Davis MH, Laureys S, Pickard JD. Detecting awareness in the vegetative state. Science 2006; 313:1402.

69. Leslie RA, James MF. Pharmacological magnetic resonance imaging: a new application for functional MRI. Trends Pharmacol Sci 2000;21:314-318.

70. Honey G, Bullmore E. Human pharmacological MRI. Trends Pharmacol Sci 2004;25:366-374.

71. Mattay VS, Callicott JH, Bertolino A, et al. Effects of dextroamphetamine on cognitive performance and cortical activation. Neuroimage 2000;12:268-275.

72. Thiel CM, Henson RN, Morris JS, Friston KJ, Dolan RJ. Pharmacological modulation of behavioral and neuronal correlates of repetition priming. J Neurosci 2001;21:6846-6852.

73. Coull JT, Nobre AC, Frith CD. The noradrenergic alpha2 agonist clonidine modulates behavioural and neuroanatomical correlates of human attentional orienting and alerting. Cereb Cortex 2001; 11:73-84.

74. Goldstein JM, Jerram M, Poldrack R, et al. Hormonal cycle modulates arousal circuitry in women using functional magnetic resonance imaging. J Neurosci 2005;25:9309-9316.

75. Goekoop R, Barkhof F, Duschek EJ, et al. Raloxifene treatment enhances brain activation during recognition of familiar items: a pharmacological fMRI study in healthy elderly males. Neuropsychopharmacology 2006;31:1508-1518.

76. Gibbs SE, D'Esposito M. Individual capacity differences predict working memory performance and prefrontal activity following dopamine receptor stimulation. Cogn Affect Behav Neurosci 2005;5:212-221. 
77. Breiter HC, Gollub RL, Weisskoff RM, et al. Acute effects of cocaine on human brain activity and emotion. Neuron 1997;19: 591-611.

78. Kalin NH, Davidson RJ, Irwin W, et al. Functional magnetic resonance imaging studies of emotional processing in normal and depressed patients: effects of venlafaxine. J Clin Psychiatry 1997; 58(suppl 16):32-39.

79. Honey GD, Bullmore ET, Soni W, Varatheesan M, Williams SC, Sharma T. Differences in frontal cortical activation by a working memory task after substitution of risperidone for typical antipsychotic drugs in patients with schizophrenia. Proc Natl Acad Sci U S A 1999;96:13432-13437.

80. Mattay VS, Tessitore A, Callicott JH, et al. Dopaminergic modulation of cortical function in patients with Parkinson's disease. Ann Neurol 2002;51:156-164.

81. Fu CH, Williams SC, Cleare AJ, et al. Attenuation of the neural response to sad faces in major depression by antidepressant treatment: a prospective, event-related functional magnetic resonance imaging study. Arch Gen Psychiatry 2004;61:877-889.

82. Davidson RJ, Irwin W, Anderle MJ, Kalin NH. The neural substrates of affective processing in depressed patients treated with venlafaxine. Am J Psychiatry 2003;160:64-75.

83. Bertolino A, Caforio G, Blasi G, et al. Interaction of COMT (Val[108/158]Met) genotype and olanzapine treatment on prefrontal cortical function in patients with schizophrenia. Am J Psychiatry 2004;161:1798-1805.

84. Schon K, Atri A, Hasselmo ME, Tricarico MD, LoPresti ML, Stern CE. Scopolamine reduces persistent activity related to longterm encoding in the parahippocampal gyrus during delayed matching in humans. J Neurosci 2005;25:9112-9123.

85. Thiel CM, Henson RN, Dolan RJ. Scopolamine but not lorazepam modulates face repetition priming: a psychopharmacological fMRI study. Neuropsychopharmacology 2002;27:282-292.

86. Thiel CM, Bentley P, Dolan RJ. Effects of cholinergic enhancement on conditioning-related responses in human auditory cortex. Eur J Neurosci 2002;16:2199-2206.

87. Thiel CM, Friston KJ, Dolan RJ. Cholinergic modulation of experience-dependent plasticity in human auditory cortex. Neuron 2002;35:567-574.

88. Bentley P, Vuilleumier P, Thiel CM, Driver J, Dolan RJ. Effects of attention and emotion on repetition priming and their modulation by cholinergic enhancement. J Neurophysiol 2003;90: 1171-1181.

89. Wink AM, Bernard F, Salvador R, Bullmore E, Suckling J. Age and cholinergic effects on hemodynamics and functional coherence of human hippocampus. Neurobiol Aging 2006;27:13951404.

90. Rombouts SA, Barkhof F, Van Meel CS, Scheltens P. Alterations in brain activation during cholinergic enhancement with rivastigmine in Alzheimer's disease. J Neurol Neurosurg Psychiatry 2002;73:665-671.

91. Saykin AJ, Wishart HA, Rabin LA, et al. Cholinergic enhancement of frontal lobe activity in mild cognitive impairment. Brain 2004;127:1574-1583.

92. Goekoop R, Rombouts SA, Jonker C, et al. Challenging the cholinergic system in mild cognitive impairment: a pharmacological fMRI study. Neuroimage 2004;23:1450-1459.

93. Goekoop R, Scheltens P, Barkhof F, Rombouts SA. Cholinergic challenge in Alzheimer patients and mild cognitive impairment differentially affects hippocampal activation - a pharmacological fMRI study. Brain 2006;129:141-157.

94. Thiel CM. Cholinergic modulation of learning and memory in the human brain as detected with functional neuroimaging. Neurobiol Learn Mem 2003;80:234-244.

95. Tsukada H, Kakiuchi T, Ando I, Shizuno H, Nakanishi S, Ouchi Y. Regulation of cerebral blood flow response to somatosensory stimulation through the cholinergic system: a positron emission tomography study in unanesthetized monkeys. Brain Res 1997; 749:10-17.

96. McGaughy J, Koene RA, Eichenbaum H, Hasselmo ME. Cholinergic deafferentation of the entorhinal cortex in rats impairs encoding of novel but not familiar stimuli in a delayed nonmatchto-sample task. J Neurosci 2005;25:10273-10281.

97. He Y, Wang L, Zang Y, et al. Regional coherence changes in the early stages of Alzheimer's disease: a combined structural and resting-state functional MRI study. Neuroimage 2007;35:488500.

98. Laufs H, Hamandi K, Salek-Haddadi A, Kleinschmidt AK, Duncan JS, Lemieux L. Temporal lobe interictal epileptic discharges affect cerebral activity in "default mode" brain regions. Hum Brain Mapp Nov 28, 2006 [Epub ahead of print].

99. Salek-Haddadi A, Diehl B, Hamandi K, et al. Hemodynamic correlates of epileptiform discharges: an EEG-fMRI study of 63 patients with focal epilepsy. Brain Res 2006;1088:148-166.

100. Fridman EA, Hanakawa T, Chung M, Hummel F, Leiguarda RC, Cohen LG. Reorganization of the human ipsilesional premotor cortex after stroke. Brain 2004;127:747-758.

101. Katscher U, Bönert P. Parellel magnetic resonance imaging. Neurotherapeutics 2007;4:498-509.

102. Lutcke H, Merboldt KD, Frahm J. The cost of parallel imaging in functional MRI of the human brain. Magn Reson Imaging 2006; $24: 1-5$.

103. Wiggins GC, Triantafyllou C, Potthast A, Reykowski A, Nittka M, Wald LL. 32-channel 3 Tesla receive-only phased-array head coil with soccer-ball element geometry. Magn Reson Med 2006; 56:216-223.

104. Kahn I, Wiggins CJ, Wiggins GC, et al. High-resolution 3T and 7T functional MRI: feasibility and specificity. Presented at the Society for Neuroscience; October 14, 2006; Atlanta.

105. Dickerson BC, Wright CI, Miller S, et al. Ultrahigh-field differentiation of medial temporal lobe function: sub-millimeter fMRI of amygdala and hippocampal activation at 7 Tesla. Presented at the Organization for Human Brain Mapping; June 12, 2006; Florence.

106. Miller KL, Smith SM, Jezzard P, Pauly JM. High-resolution FMRI at $1.5 \mathrm{~T}$ using balanced SSFP. Magn Reson Med 2006;55: 161-170.

107. Dickerson BC, Miller S, Greve DN, et al. Prefrontal-hippocampal-fusiform activity during encoding predicts intra-individual differences in free recall ability: An event-related functionalanatomic MRI study. Hippocampus; (in press).

108. Cramer SC, Crafton KR. Somatotopy and movement representation sites following cortical stroke. Exp Brain Res 2006;168:2532

109. Dickerson BC, Bakkour A, Salat DH, et al. The cortical signature of Alzheimer's disease (AD): a high-throughput in vivo MRIbased quantitative biomarker. Presented at the 20th Annual Massachusetts Alzheimer's Disease Research Center Symposium; January 11, 2007; Boston. 